\title{
Rad-supplemented Modules
}

\author{
Engin BÜyÜKașiK (*) - Engin Mermut (**) - SAlahattin ÖZdemir (***)
}

ABSTRACT - Let $\tau$ be a radical for the category of left $R$-modules for a ring $R$. If $M$ is a $\tau$-coatomic module, that is, if $M$ has no nonzero $\tau$-torsion factor module, then $\tau(M)$ is small in $M$. If $V$ is a $\tau$-supplement in $M$, then the intersection of $V$ and $\tau(M)$ is $\tau(V)$. In particular, if $V$ is a Rad-supplement in $M$, then the intersection of $V$ and $\operatorname{Rad}(M)$ is $\operatorname{Rad}(V)$. A module $M$ is $\tau$-supplemented if and only if the factor module of $M$ by $P_{\tau}(M)$ is $\tau$-supplemented where $P_{\tau}(M)$ is the sum of all $\tau$-torsion submodules of $M$. Every left $R$-module is Rad-supplemented if and only if the direct sum of countably many copies of $R$ is a Radsupplemented left $R$-module if and only if every reduced left $R$-module is supplemented if and only if $R / P(R)$ is left perfect where $P(R)$ is the sum of all left ideals $I$ of $R$ such that $\operatorname{Rad} I=I$. For a left duo ring $R, R$ is a $\operatorname{Rad}$-supplemented left $R$-module if and only if $R / P(R)$ is semiperfect. For a Dedekind domain $R$, an $R$-module $M$ is Rad-supplemented if and only if $M / D$ is supplemented where $D$ is the divisible part of $M$.

\section{Introduction.}

All rings considered in this paper will be associative with an identity element. Unless otherwise stated $R$ denotes an arbitary ring and all modules will be left unitary $R$-modules. By $R$-Mod, we denote the category of left $R$-modules. Unless otherwise stated, $\tau$ is a radical on

(*) Indirizzo dell'A.: Izmir Institute of Technology, Department of Mathematics, 35430, Urla, Izmir, Turkey.

E-mail: enginbuyukasik@iyte.edu.tr

(**) Indirizzo dell'A.: Dokuz Eylül Üniversitesi, Tinaztepe Yerleşkesi, Fen-Edebiyat Fakültesi, Matematik Bölümü, 35160, Buca/Izmir, Turkey.

E-mail: engin.mermut@deu.edu.tr

(***) Indirizzo dell'A.: Dokuz Eylül Üniversitesi, Tinaztepe Yerleşkesi, Fen-Edebiyat Fakültesi, Matematik Bölümü, 35160, Buca/Izmir, Turkey.

E-mail: salahattin.ozdemir@deu.edu.tr

1991 Mathematics Subject Classification: primary 18G25, 16D10; secondary 16S90, 16L30. 
$R$-Mod. For fundamentals on module theory, see for example [17], [4] and [30]. Let $R$ be a ring and $M$ be an $R$-module. Denote by $X \leq M$ that $X$ is a submodule of $M$. As usual, $\operatorname{Rad} M$ denotes the radical of $M$ and $J(R)$ denotes the Jacobson radical of the ring $R$. A submodule $K$ of $M$ is called small in $M$ (denoted by $K \ll M$ ) if $M \neq K+T$ for every proper submodule $T$ of $M$. For an index set $I, M^{(I)}$ denotes as usual the direct sum $\bigoplus_{i \in I} M$. The set of natural numbers is denoted by $\mathbb{N}$. See $[30, \S 41]$ and the recent monograph [10] for results (and the definitions) related to (weak) supplements and (weakly) supplemented modules. Given submodules $K \leq L \leq M$, the inclusion $K \leq L$ is called cosmall in $M$ if $L / K \ll M / K$ (see [10,3.1]). A submodule $L \leq M$ is called coclosed in $M$ if $L$ has no proper submodule $K$ for which the inclusion $K \leq L$ is cosmall in $M$ (see $[10,3.6]$ ).

We shall investigate some properties of Rad-supplemented modules and in general $\tau$-supplemented modules where $\tau$ is a radical for $R$-Mod. The motivation for considering Rad-supple-ments (coneat submodules) and $\tau$-supplements in general is given in the next section. One of the main questions we shall answer is when are all left $R$-modules Radsupplemented. In the investigation of this problem, the notion of radical modules, reduced modules and coatomic modules turn out to be useful; see [32, pp. 47]. In the definitions and properties for reduced and coatomic modules, instead of $\mathrm{Rad}$, we can use any (pre)radical $\tau$ on $R$-Mod (see Section 3), and these will be useful in the investigation of the properties of $\tau$-supplemented modules. For a module $M$, the sum of all radical submodules of $M$ is denoted by $P(M)$, that is, $P(M)$ is the sum of all submodules $U$ of $M$ such that $\operatorname{Rad} U=U$. For submodules $U$ and $V$ of a module $M$, the submodule $V$ is said to be a Rad-supplement of $U$ in $M$ or $U$ is said to have a Rad-supplement $V$ in $M$ if $U+V=M$ and $U \cap V \leq \operatorname{Rad} V$. A module $M$ is called a Rad-supplemented module if every submodule of $M$ has a Rad-supplement in $M$. See also [29]; Radsupplemented modules are called generalized supplemented modules there. In Section 6, we shall prove that every left $R$-module is Radsupplemented if and only if $R / P(R)$ is left perfect. In [9], it is proved that the class of Rad-supplemented rings lies properly between those of the semiperfect and the semilocal rings. We show that a left duo ring $R$ is Rad-supplemented as a left R-module if and only if $R / P(R)$ is semiperfect. Whenever possible the related results are given in general for a radical $\tau$ for $R$-Mod. See [1] and [10, $\S 10]$ for some properties of $\tau$ supplements and $\tau$-supplemented modules. We shall investigate some 
further properties of $\tau$-supplemented modules in Section 4. For some rings $R$, we shall also determine when all left $R$-modules are $\tau$-supplemented in Section 5. We are also going to study the property $\operatorname{Rad} V=V \cap \operatorname{Rad} M$ for a submodule $V$ of $M$. It is known that this holds if $V$ is a supplement in $M$ (see [30,41.1]) and moreover if $V$ is coclosed in $M$ (see [10, 3.7]). We show that this property also holds when $V$ is a Radsupplement in $M$ (Corollary 4.2); in general for a radical $\tau$ for $R$-Mod, we show that if $V$ is a $\tau$-supplement in $M$, then $\tau(V)=V \cap \tau(M)$. It is clear that every supplemented module is Rad-supplemented. But the converse implication fails to be true. For example, the Z $\mathrm{Z}$-module $\mathrm{Q}$ is Radsupplemented but not supplemented. Since $\operatorname{Rad} Q=Q$ (see for example [17, 2.3.7]), $Q$ is Rad-supplemented (by Proposition 4.5-(i)). But $Q$ is not supplemented by example [10, 20.12]. In Section 7 , we understand this example clearly and describe Rad-supplemented modules over Dedekind domains using the structure of supplemented modules over Dedekind domains which was completely determined in [32].

For definitions and elementary properties of preradicals, see [26, Ch. VI], [6] or [10, § 6]. A preradical $\tau$ for $R-\operatorname{Mod}$ is defined to be a subfunctor of the identity functor on $R-\mathcal{M} o d$. Let $\tau$ be a preradical for $R-\mathcal{M} o d$. The following module classes are defined: the preradical or (pre)torsion class of $\tau$ is

$$
\mathbb{T}_{\tau}=\{N \in R-\mathcal{M o d} \mid \tau(N)=N\}
$$

and the preradical free or (pre)torsion free class of $\tau$ is

$$
\mathrm{F}_{\tau}=\{N \in R-\mathcal{M o d} \mid \tau(N)=0\}
$$

$\tau$ is said to be idempotent if $\tau(\tau(N))=\tau(N)$ for every $R$-module $N$. $\tau$ is said to be a radical if $\tau(N / \tau(N))=0$ for every $R$-module $N$. For the main elementary properties that we shall use frequently for a (pre)radical, see for example [10, pp. 55]. For $R$-modules $K \leq M$, we always have $(\tau(M)+K) / K \leq \tau(M / K)$. If moreover $\tau$ is a radical and $K \leq \tau(M)$, then $\tau(M / K)=\tau(M) / K$ [26, Ch. VI, Lemma 1.1]. When we consider a ring $R$ as a left $R$-module, we already have that $A=\tau\left({ }_{R} R\right)$ is a left ideal of $R$; indeed it is a two-sided ideal of $R$ [26, Ch. VI, § 1, Examples (3), pp. 139] so that we can consider the quotient ring $R / A$ which we shall use in the results for $\tau$-supplemented modules. For a free $R$-module $F$, the property $\tau(F)=\tau(R) F$ is easily obtained. This also holds for projective modules. See also [13] and [7] for some related concepts in torsion theories (mostly for a hereditary preradical). 


\section{Coneat submodules and Rad-supplements.}

Neat subgroups of abelian groups (introduced in [15, pp. 43-44]) have been generalized to modules in $[28,9.6]$ (and $[27, \S 3]$ ). The class of coneat submodules has been introduced in [21] and [3]: A monomorphism $f: K \rightarrow L$ is called coneat if each module $M$ with $\operatorname{Rad} M=0$ is injective with respect to it, that is, the Hom sequence

$$
\operatorname{Hom}(L, M) \rightarrow \operatorname{Hom}(K, M) \rightarrow 0
$$

is exact. See [21, Proposition 3.4.2] or [10, 10.14] or [1, 1.14] for a characterization of coneat submodules. This characterization will be the particular case $\tau=$ Rad in Proposition 2.1 and this is the reason for considering Rad-supplements and in general $\tau$-supplements given below. For more results on coneat submodules see [21], [3], [10, § 10 and 20.7-8], [1] and [24].

Proper classes of monomorphisms and short exact sequences were introduced in [8] to do relative homological algebra. In [27, Remark after Proposition 6], it is pointed out that supplement submodules induce a proper class of short exact sequences (the term 'low' is used for supplements dualizing the term 'high' used in abelian groups). [12] uses the terminology 'cohigh' for supplements and gives more general definitions for proper classes of supplements related to another given proper class (motivated by the considerations as pure-high extensions and neat-high extensions in [14]). For the definition and properties of proper classes, see [25], [20, Ch. 12, § 4], [28] and [22]. We shall follow the terminology and notation as in [10, § 10] and [1] since we will mainly refer to these for $\tau$ supplemented modules and Rad-supplemented modules.

Denote by $\mathbb{E}_{\text {Suppl }}$ the class of all short exact sequences induced by supplement submodules; that is $\mathbb{E}_{\text {Suppl }}$ is the class of all short exact sequences

$$
0 \longrightarrow A \stackrel{f}{\longrightarrow} B \stackrel{g}{\longrightarrow} C \longrightarrow 0
$$

of $R$-modules and $R$-module homomorphisms such that $\operatorname{Im}(f)$ is a supplement in $B$. Then as mentioned above, the class $\mathbb{E}_{\text {Suppl }}$ forms a proper class, see for example [10, 20.7]. Every module $M$ with $\operatorname{Rad} M=0$ is $\mathbb{E}_{S u p p l}$-injective that is $M$ is injective with respect to every short exact sequence in $\mathbb{E}_{\text {Suppl }}$. Thus supplement submodules are coneat submodules by the definition of coneat submodules. In the definition of coneat submodules, using any radical $\tau$ instead of Rad, the following result is obtained. It gives us the definition of a $\tau$-supplement in a module because the last condition is like 
the usual supplement condition except that, instead of $U \cap V \ll V$, the condition $U \cap V \leq \tau(V)$ is required.

Proposition 2.1 (see $[10,10.11]$ or $[1,1.11]$ ). Let $\tau$ be a radical for $R$-Mod. For a submodule $V \leq M$, the following statements are equivalent.

(i) Every module $N$ with $\tau(N)=0$ is injective with respect to the inclusion $V \hookrightarrow M$;

(ii) there exists a submodule $U \leq M$ such that

$$
U+V=M \text { and } U \cap V=\tau(V) ;
$$

(iii) there exists a submodule $U \leq M$ such that

$$
U+V=M \text { and } U \cap V \leq \tau(V) .
$$

If these conditions are satisfied, then $V$ is called a $\tau$-supplement in $M$.

The usual definitions are then given as follows. For submodules $U$ and $V$ of a module $M$, the submodule $V$ is said to be a $\tau$-supplement of $U$ in $M$ or $U$ is said to have a $\tau$-supplement $V$ in $M$ if $U+V=M$ and $U \cap V \leq \tau(V)$. A module $M$ is called a $\tau$-supplemented module if every submodule of $M$ has a $\tau$-supplement in $M$. We call $M$ totally $\tau$-supplemented if every submodule of $M$ is $\tau$-supplemented. A submodule $N$ of $M$ is said to have ample $\tau$-supplements in $M$ if for every $L \leq M$ with $N+L=M$, there is a $\tau$-supplement $L^{\prime}$ of $N$ with $L^{\prime} \leq L$. A module $M$ is said to be amply $\tau$-supplemented if every submodule of $M$ has ample $\tau$-supplements in $M$.

For $\tau=\operatorname{Rad}$, the above definitions give Rad-supplement submodules of a module, Rad-supplemented modules, etc. By these definitions, a submodule $V$ of a module $M$ is a coneat submodule of $M$ if and only if $V$ is a Rad-supplement of a submodule $U$ of $M$ in $M$.

\section{3. $\tau$-reduced and $\tau$-coatomic modules, and the largest $\tau$-torsion submodule $\boldsymbol{P}_{\tau}(M)$.}

Let $\tau$ be a preradical for $R$-Mod and let $M$ be an $R$-module. By taking $\tau$ instead of Rad in the definitions of reduced and coatomic module definitions in [32, pp. 47], we define the following:

(i) $M$ is said to be a $\tau$-torsion module if $\tau(M)=M$, that is $M$ is in the pretorsion class ${ }^{~} T_{\tau}$. 
(ii) By $P_{\tau}(M)$ we denote the sum of all $\tau$-torsion submodules of $M$, that is,

$$
P_{\tau}(M)=\sum\{U \leq M \mid \tau(U)=U\} .
$$

(iii) $M$ is said to be a $\tau$-reduced module if it has no nonzero $\tau$-torsion submodule, that is, for every submodule $U$ of $M, \tau(U)=U$ implies $U=0$; equivalently, $\tau(U) \neq U$ for every nonzero submodule $U$ of $M$. Clearly, $M$ is $\tau$-reduced if and only if $M$ is $P_{\tau}$-torsion free, that is, $P_{\tau}(M)=0$.

(iv) $M$ is said to be a $\tau$-coatomic module if it has no nonzero $\tau$-torsion factor module, that is, for every submodule $U$ of $M, \tau(M / U)=M / U$ implies $U=M$; equivalently, $\tau(M / U) \neq M / U$ for every proper submodule $U$ of $M$.

For $\tau=\operatorname{Rad}, P_{\tau}(M)$ will be denoted by just $P(M)$, a Rad-torsion module is called a radical module, a Rad-reduced module will be called a reduced module and a Rad-coatomic module will be called a coatomic module following the terminology in [32]. Coatomic modules appear in the theory of supplemented, semiperfect, and perfect modules. See [32, Lemma 1.5] for some properties of reduced and coatomic modules. For the structure of coatomic modules over commutative Noetherian rings see [33]; the Noetherian assumption is needed to have that every submodule of a coatomic module over a commutative Noetherian ring is coatomic [33, Lemma 1.1].

For completeness note the following elementary properties of $P_{\tau}(M)$ :

THeOREm 3.1. Let $\tau$ be a preradical for $R$-Mod and let $M$ be an $R$ module.

(i) $P_{\tau}$ is an idempotent preradical.

(ii) If $M \leq N$ for a module $N$, then $P_{\tau}(M) \leq \tau(N)$. In particular, $P_{\tau}(M) \leq \tau(M)$.

(iii) $\tau\left(P_{\tau}(M)\right)=P_{\tau}(M)$, that is, $P_{\tau}(M)$ is $\tau$-torsion, and so by its definition $P_{\tau}(M)$ is the largest $\tau$-torsion submodule of $M$.

(iv) If $P_{\tau}(M) \leq V$ for a submodule $V$ of $M$, then $P_{\tau}(M) \leq \tau(V)$.

(v) $P_{\tau}(\tau(M))=P_{\tau}(M)$

(vi) The pretorsion class of $P_{\tau}$ equals the pretorsion class of $\tau$ and the pretorsion free class of $P_{\tau}$ contains the pretorsion free class of $\tau$ :

$$
{ }^{\top} \mathbb{T}_{P_{\tau}}=\mathbb{T}_{\tau} \quad \text { and } \quad \mathbb{F}_{P_{\tau}} \supseteq \mathbb{F}_{\tau} .
$$

(vii) Moreover, if $\tau$ is a radical, then the factor module $M / P_{\tau}(M)$ is $\tau$-reduced, that is, $P_{\tau}\left(M / P_{\tau}(M)\right)=0$ and so $P_{\tau}$ is an idempotent radical. 
REMARK 3.2. In general, given any class $\mathrm{A}$ of modules, a preradical $\tau^{\mathrm{A}}$ is defined by setting for each module $N$,

$$
\tau^{\mathrm{A}}(N)=\sum\{\operatorname{Im} f \mid f: A \rightarrow N \text { in } R-\mathcal{M} \text { od, } A \in \mathrm{A}\} .
$$

and if $\mathrm{A}$ is a pretorsion class, then $\tau^{\mathrm{A}}$ is an idempotent preradical (see for example [10, 6.5-6]). In our case, the preradical $P_{\tau}$ is equal to $\tau^{\mathrm{A}}$ when the pretorsion class $\mathrm{A}=T_{\tau}$, the torsion class of $\tau$. See also [26, Ch. VI, $\S 1$ ]; $P_{\tau}$ is the largest idempotent preradical that is smaller than $\tau$ and see [26, Ch. VI, Exercise 4, p. 157] for the properties Theorem 3.1-(iii,v). Since $P_{\tau}$ is an idempotent radical when $\tau$ is a radical, it gives a torsion theory for $R$-Mod with torsion class ${ }^{2} T_{P_{\tau}}=\mathbb{T}_{\tau}$ and torsion free class $\mathrm{F}_{P_{\tau}}$. By the results in [26, Ch. VI, $\S 2]$, the properties for $\tau$-torsion and $\tau$-reduced modules in the following Proposition 3.4 are obtained because $\tau$-torsion modules equate with $P_{\tau}$-torsion modules and $\tau$-reduced modules form the torsion free class $\mathbb{F}_{P_{\tau}}$.

REMARK 3.3. See [13, pp. 29, 63] for the definitions and properties of $\tau$ dense submodules of a module and $\tau$-cotorsionfree modules for a hereditary idempotent preradical $\tau$ on $R$-Mod: A submodule $N$ of a module $M$ is said to be $\tau$-dense in $M$ if $M / N$ is $\tau$-torsion, that is, $\tau(M / N)=M / N$, and a module $M$ is said to be $\tau$-cotorsionfree if it has no proper $\tau$-dense submodules. Our definition of $\tau$-coatomic module coincides with $\tau$-cotorsionfree module but in our case, $\tau$ need not be idempotent or hereditary. Observe that since being $\tau$-torsion is the same with being $P_{\tau}$-torsion and $P_{\tau}$ is an idempotent preradical, the idempotent assumption is not a problem. But in our case $\tau$ is not assumed to be hereditary; in particular, Rad is not hereditary. The properties for $\tau$-cotorsionfree modules given in [13] hold under this hereditary assumption. For example, arbitary direct sum of $\tau$ cotorsionfree modules is $\tau$-cotorsionfree when $\tau$ is a hereditary idempotent preradical but in our case, for just an (idempotent) preradical $\tau$, arbitrary direct sum of $\tau$-coatomic modules need not be $\tau$-coatomic.

Note also the following properties of $\tau$-reduced and $\tau$-coatomic modules which are easily proved:

Proposition 3.4. Let $\tau$ be a preradical for R-Mod.

(i) The class of $\tau$-torsion modules is closed under quotients and direct sums. Moreover, if $\tau$ is a radical, then the class of $\tau$-torsion modules is closed under extensions. 
(ii) The class of $\tau$-reduced modules is closed under submodules, direct products and direct sums.

(iii) Every factor module of a $\tau$-coatomic module is $\tau$-coatomic.

(iv) The class of $\tau$-reduced, respectively $\tau$-coatomic, modules is closed under extensions, that is, if

$$
0 \longrightarrow A \longrightarrow B \longrightarrow C \longrightarrow 0
$$

is a short exact sequence of modules such that $A$ and $C$ are $\tau$-reduced, respectively $\tau$-coatomic, then $B$ is also $\tau$-reduced, respectively $\tau$-coatomic.

Proposition 3.5. Let $\tau$ be a radical for $R$-Mod. If a module $M$ is $\tau$ coatomic, then $\tau(M) \ll M$.

Proof. Suppose $\tau(M)+L=M$ for some submodule $L \leq M$. Since $M / L=(\tau(M)+L) / L \leq \tau(M / L)$, we obtain $M / L=\tau(M / L)$. This gives $L=M$ since $M$ is $\tau$-coatomic. Hence $\tau(M) \ll M$.

\section{4. $\tau$-supplemented modules.}

Throughout the rest of the paper, $\tau$ denotes a radical on $R$-Mod (where $R$ is an arbitrary ring). See [1] and [10, $\S 10]$ for properties of $\tau$-supplements and $\tau$-supplemented modules. In this section, we shall see some other properties of $\tau$-supplemented modules. We shall frequently use the fact that any factor module of a $\tau$-supplemented module is $\tau$-supplemented $[1,2.2(2)]$.

THEOREM 4.1. If $V$ is a $\tau$-supplement in a module $M$, then $\tau(V)=V \cap \tau(M)$.

Proof. $\tau(V) \leq V \cap \tau(M)$ always holds. To show the converse we only require to show that $(V \cap \tau(M)) / \tau(V)=0$. Since $V$ is a $\tau$-supplement in $M$, there exists a submodule $U \leq M$ such that $U+V=M$ and $U \cap V=\tau(V)$ by Proposition 2.1-(ii)). Then

$$
M /(U \cap V)=(U /(U \cap V)) \oplus((V / U \cap V))=(U / \tau(V) \oplus(V / \tau(V)) .
$$

Since $\tau$ is a radical, we obtain:

$$
\tau(M / \tau(V))=\tau(U / \tau(V)) \oplus \tau(V / \tau(V))=\tau(U / \tau(V)) \oplus 0=\tau(U / \tau(V)) .
$$


By properties of a radical, since $\tau(V) \leq \tau(M)$, we have:

$$
\begin{aligned}
& \tau(M) / \tau(V)=\tau(M / \tau(V))=\tau(U / \tau(V)), \quad \text { and } \\
& (V \cap \tau(M)) / \tau(V)=(V / \tau(V)) \cap(\tau(M) / \tau(V))=(V / \tau(V)) \cap \tau(U / \tau(V)) \\
& \leq(V / \tau(V)) \cap(U / \tau(V)) \\
& =(U \cap V) / \tau(V)=\tau(V) / \tau(V)=0 .
\end{aligned}
$$

COROLlary 4.2. If $V$ is a Rad-supplement in a module $M$, then

$$
\operatorname{Rad} V=V \cap \operatorname{Rad} M .
$$

Proposition 4.3. Let $K, L, M$ be modules such that $K \leq L \leq M$.

(i) If $K$ is a $\tau$-supplement in $M$, then it is a $\tau$-supplement in $L$.

(ii) If $K \leq \tau(L)$ and $L / K$ is a $\tau$-supplement in $M / K$, then $L$ is a $\tau$-supplement in $M$.

(iii) If $K$ is a $\tau$-supplement in $L$ and $L$ is a $\tau$-supplement in $M$, then $K$ is a $\tau$-supplement in $M$.

Proof. (i) Since $K$ is a $\tau$-supplement in $M$, there exists a submodule $U \leq M$ such that $U+K=M$ and $U \cap K \leq \tau(K)$. So $L=L \cap M$ $=L \cap(U+K)=L \cap U+K$ and $(L \cap U) \cap K=U \cap K \leq \tau(K)$.

(ii) Since $L / K$ is a $\tau$-supplement in $M / K$, there exists a submodule $U \leq M$ with $K \leq U$ such that $U / K+L / K=M / K$ and $(U / K) \cap(L / K)$ $\leq \tau(L / K)$. So we obtain $U+L=M$ and

$$
(U \cap L) / K=(U / K) \cap(L / K) \leq \tau(L / K)=\tau(L) / K
$$

by properties of a radical since $K \leq \tau(L)$. Hence $U \cap L \leq \tau(L)$ and so $L$ is a $\tau$-supplement (of $U$ ) in $M$.

(iii) Temporarily denote by $\mathbb{E}$ the class induced by $\tau$-supplement submodules; that is $\mathbb{E}$ is the class of all short exact sequences

$$
0 \longrightarrow A \stackrel{f}{\longrightarrow} B \stackrel{g}{\longrightarrow} C \longrightarrow 0
$$

of $R$-modules and $R$-module homomorphisms such that $\operatorname{Im}(f)$ is a $\tau$-supplement in $B$. For such a short exact sequence in the class $\mathbb{E}, f$ is said to be an $\mathbb{E}$-monomorphism. By Proposition 2.1, the class $\mathbb{E}$ is the proper class injectively generated by all modules $M$ such that $\tau(M)=0$. By the definition of proper classes, the composition of two $\mathbb{E}$-monomorphisms is an $\mathbb{E}$ monomorphism (see [10, 10.1]). If $K$ is a $\tau$-supplement in $L$ and $L$ is a $\tau$ supplement in $M$, then the inclusions $K \hookrightarrow L$ and $L \hookrightarrow M$ are $\mathbb{E}$-mono- 
morphisms and so their composition $K \hookrightarrow M$ is also an $\mathbb{E}$-monomorphism, that is, $K$ is a $\tau$-supplement in $M$.

Proposition 4.4. Let $M$ be a module and let $N, K$ be submodules of $M$ such that $M=N+K$. If $K$ is $\tau$-supplemented, then $K$ contains a $\tau$-supplement of $N$ in $M$.

Proof. Since $K$ is $\tau$-supplemented, the submodule $N \cap K$ of $K$ has a $\tau$-supplement in $K$, that is, there exists a submodule $L \leq K$ such that $(N \cap K)+L=K$ and $(N \cap K) \cap L \leq \tau(L)$. Then $M=N+K=N$ $+(N \cap K)+L=N+L$ and $N \cap L=(N \cap K) \cap L \leq \tau(L)$. Hence $L$ is a $\tau$-supplement of $N$ in $M$.

It is trivial to show that:

\section{Proposition 4.5.}

(i) Every $\tau$-torsion module is $\tau$-supplemented.

(ii) The module $P_{\tau}(M)$ is $\tau$-supplemented for every module $M$.

THeOREM 4.6. If a module $M$ is $\tau$-reduced and $\tau$-supplemented, then $M$ is $\tau$-coatomic, $\operatorname{Rad} M=\tau(M)$ and $M$ is weakly supplemented.

Proof. Let $U$ be a proper submodule of $M$. Since $M$ is $\tau$-supplemented, there exists a submodule $V \leq M$ such that $U+V=M$ and $U \cap V \leq \tau(V)$. So we have $\tau(V /(U \cap V))=\tau(V) /(U \cap V)$ by properties of a radical. We also have $\tau(V) \neq V$ since $M$ is $\tau$-reduced, and so $\tau(V) /(U \cap V) \neq V /(U \cap V)$. Therefore, using the fact that $M / U=(U+V) / U \cong V /(U \cap V)$ we obtain

$$
\tau(M / U) \cong \tau(V /(U \cap V))=\tau(V) /(U \cap V) \neq V /(U \cap V),
$$

or equivalently, $\tau(M / U) \neq M / U$, that is, $M$ is $\tau$-coatomic. By Proposition 3.5, $\tau(M) \ll M$ and hence $\tau(M) \leq \operatorname{Rad} M$. By [1, 2.2(3)], $M / \tau(M)$ is semisimple since $M$ is $\tau$-supplemented. Then $\operatorname{Rad}(M / \tau(M))=0$ and so $\operatorname{Rad} M \leq \tau(M)$. Thus $\operatorname{Rad} M=\tau(M)$. Since $\operatorname{Rad} M=\tau(M) \ll M$ and $M$ is a semilocal module (that is $M / \operatorname{Rad} M=M / \tau(M)$ is semisimple), we obtain that $M$ is weakly supplemented by [19, Theorem 2.7].

TheOREM 4.7. If $M$ is a $\tau$-supplemented module, then $\operatorname{Rad} M \leq \tau(M)$, and

$$
\operatorname{Rad}\left(M / P_{\tau}(M)\right)=\tau\left(M / P_{\tau}(M)\right)=\tau(M) / P_{\tau}(M)
$$


Proof. By [1, 2.2(3)], $M / \tau(M)$ is semisimple and so $\operatorname{Rad}(M / \tau(M))=0$ which gives $\operatorname{Rad} M \leq \tau(M)$. The module $M / P_{\tau}(M)$ is $\tau$-supplemented as a factor module of the $\tau$-supplemented module $M$. Since $M / P_{\tau}(M)$ is $\tau$-reduced, $\operatorname{Rad}\left(M / P_{\tau}(M)\right)=\tau\left(M / P_{\tau}(M)\right)$ by Theorem 4.6. By properties of a radical, $\tau\left(M / P_{\tau}(M)\right)=\tau(M) / P_{\tau}(M)$.

Proposition 4.8. The following are equivalent for a module $M$ and $a$ submodule $K \leq P_{\tau}(M)$ :

(i) $M$ is $\tau$-supplemented;

(ii) $M / K$ is $\tau$-supplemented;

(iii) $M / P_{\tau}(M)$ is $\tau$-supplemented.

Proof. Since every factor module of a $\tau$-supplemented module is $\tau$-supplemented, (i) $\Rightarrow$ (ii) $\Rightarrow$ (iii) are clear. To prove (iii) $\Rightarrow$ (i), take $U \leq M$. By hypothesis, there is a submodule $V \leq M$ such that $P_{\tau}(M) \leq V$,

$$
\left[\left(U+P_{\tau}(M)\right) / P_{\tau}(M)\right]+\left[V / P_{\tau}(M)\right]=M / P_{\tau}(M)
$$

and

$$
\begin{aligned}
\left(U \cap V+P_{\tau}(M)\right) / P_{\tau}(M) & =\left[\left(U+P_{\tau}(M)\right) / P_{\tau}(M)\right] \cap\left[V / P_{\tau}(M)\right] \\
& \leq \tau\left(V / P_{\tau}(M)\right)=\tau(V) / P_{\tau}(M) .
\end{aligned}
$$

Note that the last equality holds by Theorem 3.1-(iv). So we have $U+V=M$ and $U \cap V \leq \tau(V)$. That is $V$ is a $\tau$-supplement of $U$ in $M$.

COROLLARY 4.9. The following are equivalent for a ring $R$ :

(i) every R-module is $\tau$-supplemented;

(ii) every free $R$-module is $\tau$-supplemented;

(iii) every $\tau$-reduced $R$-module is $\tau$-supplemented.

Proof. (i) $\Rightarrow$ (ii) and (i) $\Rightarrow$ (iii) are clear. (ii) $\Rightarrow$ (i) follows since every module is an epimorphic image of a free $R$-module and being $\tau$-supplemented is preserved under passage factor modules. To prove (iii) $\Rightarrow$ (i) take an $R$-module $M$. Since $M / P_{\tau}(M)$ is $\tau$-reduced, we obtain that $M / P_{\tau}(M)$ is $\tau$-supplemented by the hypothesis. So $M$ is $\tau$-supplemented by Proposition 4.8 .

Proposition 4.10. If $V$ is a $\tau$-supplement in a module $M$ and $V$ is $\tau$-coatomic, then $V$ is a supplement in $M$. 
Proof. Since $V$ is a $\tau$-supplement in $M$, there exists $U \leq M$ such that $U+V=M$ and $U \cap V \leq \tau(V)$. Since $V$ is $\tau$-coatomic, we have by Proposition 3.5 that $\tau(V) \ll V$. Then $U \cap V \leq \tau(V) \ll V$ and so $V$ is a supplement in $M$.

Proposition 4.11. If $M$ is a $\tau$-reduced module that is totally $\tau$-supplemented, then $M$ is totally supplemented.

Proof. Since being $\tau$-reduced is inherited by submodules, it is enough to prove that $M$ is supplemented. Let $U \leq M$ and $V$ be a $\tau$-supplement of $U$ in $M$. Then $U+V=M$ and $U \cap V \leq \tau(V)$. By hypothesis, $V$ is $\tau$-supplemented and $\tau$-reduced. So by Theorem 4.6, $V$ is $\tau$-coatomic. Then $\tau(V) \ll V$ by Proposition 3.5. Therefore $U \cap V \ll V$ and so $V$ is a supplement of $U$ in $M$. Hence $M$ is supplemented.

Clearly supplemented modules are Rad-supplemented and so we obtain the following:

COROLLARY 4.12. If $M$ is a reduced module, then $M$ is totally Radsupplemented if and only if $M$ is totally supplemented.

\section{When are all left $R$-modules $\tau$-supplemented?}

In this section, we shall characterize the rings all of whose (left) modules are $\tau$-supplemented for some particular radicals $\tau$ including Rad.

An epimorphism $f: P \rightarrow M$ is said to be a projective cover if $P$ is projective and $\operatorname{Ker} f \ll P$. A property that we shall use is that if $P$ is projective and $P / U$ has a projective cover, then $U$ has a supplement $V$ in $P$ such that $V$ is a direct summand of $P$ and hence projective (see [30, 42.1]). A ring $R$ is called left perfect if every left $R$-module has a projective cover. Recall that, a subset $I$ of a ring $R$ is said to be left T-nilpotent in case for every sequence $\left\{a_{k}\right\}_{k=1}^{\infty}$ in $I$ there is a positive integer $n$ such that $a_{1} \cdots a_{n}=0$. A ring $R$ is said to be a left max ring if every left $R$-module has a maximal submodule, equivalently $\operatorname{Rad}(M) \ll M$ for every left $R$-module $M$. A ring $R$ is said to be a semilocal ring if $R / J(R)$ is a semisimple ring (that is a left (and right) semisimple $R$-module), see [18, § 20]. Semilocal rings are also referred to as rings semisimple modulo their radical (see $[4, \S 15$, pp. 170172]). For a semilocal ring $R, \operatorname{Rad} M=J M$ for every left $R$-module $M$ where $J=J(R)$ (see for example [4, Corollary 15.18]). By a characteriza- 
tion of left perfect rings by Bass, as in for example [4, Theorem 28.4], a ring $R$ is left perfect if and only if $R$ is a semilocal ring and $J(R)$ is left T-nilpotent if and only if $R$ is a semilocal left max ring. A ring $R$ is called left semiperfect if every finitely generated left $R$-module has a projective cover. A ring $R$ is (left or right) semiperfect if and only if the left (or right) $R$-module $R$ is supplemented (see [30, 42.6]).

An epimorphism $f: N \rightarrow M$ is said to be a $\tau$-cover if $\operatorname{Ker} f \leq \tau(N)$. If moreover $N$ is projective, then $f$ is called a projective $\tau$-cover. A ring $R$ is called left $\tau$-perfect if every left $R$-module has a projective $\tau$-cover. These rings are studied in [5] and [31] for the radical $\tau=\operatorname{Rad}$, and in [23] for a larger class of preradicals. A ring $R$ is called left $\tau$-semiperfect if every finitely generated left $R$-module has a projective $\tau$-cover. The relation between $\tau$-cover and $\tau$-supplements is the following:

Proposition 5.1 [1, 2.14]. For an R-module $L$ and $U \leq L$, the following are equivalent:

(i) $L / U$ has a projective $\tau$-cover;

(ii) $U$ has a $\tau$-supplement $V$ which has a projective $\tau$-cover.

It is clear from the definitions and Proposition 5.1 that, if $R$ is a left $\tau$ (semi)perfect ring then every (finitely generated) left $R$-module is $\tau$-supplemented. But the converse need not be true, for example when $\tau=\operatorname{Rad}$; see Example 6.2.

LEMMA 5.2. If $R$ is a ring that is a $\tau$-reduced left $R$-module and if the free left $R$-module $F=R^{(\mathrm{N})}$ is $\tau$-supplemented, then $\tau(R)$ is left T-nilpotent.

Proof. Since $P_{\tau}(R)=0$ and $P_{\tau}(F)=\left(P_{\tau}(R)\right)^{(\mathrm{N})}=0, F$ is $\tau$-reduced. Then $F$ is $\tau$-coatomic by Theorem 4.6, and so by Proposition 3.5

$$
\tau(R) F=(\tau(R))^{(\mathbb{N})}=\tau(F) \ll F .
$$

Therefore $\tau(R)$ is left $T$-nilpotent by [4, Lemma 28.3].

THEOREM 5.3. If $R$ is a ring that is a $\tau$-reduced left $R$-module, then the free left $R$-module $F=R^{(\mathrm{N})}$ is $\tau$-supplemented if and only if $R$ is left perfect and $\tau(R)=J(R)$.

Proof. Suppose $F=R^{(\mathrm{N})}$ is $\tau$-supplemented. Then $R$ is $\tau$-supplemented as a direct summand of $F$. Since $R$ is also $\tau$-reduced by hypothesis, we obtain $\tau(R)=J(R)$ by Theorem 4.6. By Lemma 5.2, $J(R)=\tau(R)$ is left 
$T$-nilpotent. Since $R$ is $\tau$-supplemented, $R / J(R)=R / \tau(R)$ is semisimple by $[1,2.2(3)]$. Hence $R$ is left perfect by [4, Theorem 28.4]. Conversely suppose $R$ is left perfect and $\tau(R)=J(R)$. Let $U \leq F=R^{(\mathrm{N})}$. Since $R$ is left perfect, every left $R$-module, and in particular, $F / U$ has a projective cover. Then by [30,42.1]), $U$ has a supplement $V$ in the free module $F$ such that $V$ is a direct summand of $F$. Since $F$ is free, its direct summand $V$ is projective. So $\tau(V)=\tau(R) V$ by properties of radicals. Since $V$ is a supplement of $U$ in $M$, $U+V=M$ and $U \cap V \ll V$. So $U \cap V \leq \operatorname{Rad}(V)$. Since $R$ is a left perfect ring, it is a semilocal ring and so $\operatorname{Rad}(V)=J(R) V$. Thus $U \cap V \leq \operatorname{Rad}(V)$ $=J(R) V=\tau(R) V=\tau(V)$. Hence $V$ is a $\tau$-supplement of $U$ in $M$.

Note that the above proof for the converse implication works for every free left $R$-module $F$, not necessarily countably generated. Moreover, since every factor module of a $\tau$-supplemented module is $\tau$-supplemented and every module is isomorphic to a factor module of a free module, we have:

COROLLARY 5.4. If $R$ is a ring that is a $\tau$-reduced left $R$-module, then every (free) left $R$-module is $\tau$-supplemented if and only if $R$ is left perfect and $\tau(R)=J(R)$.

It is easy to see that a radical $\tau$ on $R$-modules is also a radical on $R / P_{\tau}(R)$-modules since every $R / P_{\tau}(R)$-module can be considered as an $R$ module (with annihilator containing $P_{\tau}(R)$ ). We shall use this fact in the proof of the following theorem:

THeorem 5.5. For a ring $R$ with $P_{\tau}(R) \leq J(R)$, the following are equivalent.

(i) every left $R$-module is $\tau$-supplemented;

(ii) every free left $R$-module is $\tau$-supplemented;

(iii) the free left $R$-module $F=R^{(\mathrm{N})}$ is $\tau$-supplemented;

(iv) the quotient ring $R / P_{\tau}(R)$ is left perfect and $\tau(R)=J(R)$.

Proof. (i) $\Leftrightarrow$ (ii) follows by Corollary 4.9. (ii) $\Rightarrow$ (iii) is clear.

(iii) $\Rightarrow$ (iv): Since $F$ is $\tau$-supplemented, so is its factor module $\bar{F}=F / P_{\tau}(F)$ $\cong\left(R / P_{\tau}(R)\right)^{(\mathbb{N})}$. The $R$-module $\bar{F}$ can be considered as an $R / P_{\tau}(R)$-module and $\tau$ can be considered also as a radical on $R / P_{\tau}(R)$-modules. By Theorem 5.3, since $R / P_{\tau}(R)$ is $\tau$-reduced, we obtain that the quotient ring $R / P_{\tau}(R)$ is left perfect and

$$
\tau\left(R / P_{\tau}(R)\right)=J\left(R / P_{\tau}(R)\right)
$$


Then by properties of radicals, $\tau\left(R / P_{\tau}(R)\right)=\tau(R) / P_{\tau}(R)$ and $J\left(R / P_{\tau}(R)\right)$ $=J(R) / P_{\tau}(R)$ since $P_{\tau}(R) \leq J(R)$ by hypothesis. Hence $\tau(R)=J(R)$.

(iv) $\Rightarrow$ (ii): By properties of radicals, since $P_{\tau}(R) \leq \tau(R)=J(R)$ by hypothesis, we obtain for the left perfect quotient ring $S=R / P_{\tau}(R)$ that:

$$
\tau(S)=\tau\left(R / P_{\tau}(R)\right)=\tau(R) / P_{\tau}(R)=J(R) / P_{\tau}(R)=J\left(R / P_{\tau}(R)\right)=J(S) .
$$

By Corollary 5.4, every free $S$-module is $\tau$-supplemented, where we consider $\tau$ also as a radical on $S$-modules. Let $F$ be a free $R$-module. Then $F \cong R^{(I)}$ for some index set $I$. By Proposition 4.8, it is enough to prove that $\bar{F}=F / P_{\tau}(F) \cong S^{(I)}$ is $\tau$-supplemented. But this holds since $\bar{F}$ can be considered as a free $S$-module.

\section{When are all left $R$-modules Rad-supplemented?}

Using the results of the previous sections for $\tau=\operatorname{Rad}$, we obtain the following characterization of the rings $R$ over which every $R$-module is Rad-supplemented. Of course, more work still remains to understand $P(R)$ and the condition that $R / P(R)$ is left perfect.

\section{THeorem 6.1. For a ring $R$, the following are equivalent.}

(i) every left $R$-module is Rad-supplemented;

(ii) every reduced left $R$-module is Rad-supplemented;

(iii) every reduced left $R$-module is supplemented;

(iv) the free left $R$-module $R^{(\mathrm{N})}$ is Rad-supplemented;

(v) $R / P(R)$ is left perfect.

Proof. (i) $\Leftrightarrow$ (iv) $\Leftrightarrow$ (v) is obtained by Theorem 5.5 since $P(R)$ $\leq \operatorname{Rad}(R)=J(R)$. (i) $\Leftrightarrow$ (ii) follows by Corollary 4.9. (iii) $\Rightarrow$ (ii) holds since supplemented modules are Rad-supplemented. To prove (ii) $\Rightarrow$ (iii), take any reduced left $R$-module $M$. Then every submodule of $M$ is also reduced and Rad-supplemented by hypothesis (ii). So $M$ is a reduced module that is totally Rad-supplemented. By Corollary 4.12, $M$ is totally supplemented and hence supplemented.

The following is an example of a ring $R$ that is not left perfect (and so not left Rad-perfect by [23, Theorem 1.5]) but where all $R$-modules are Rad-supplemented. 
ExAmple 6.2. Let $k$ be a field. In the polynomial ring $k\left[x_{1}, x_{2}, \ldots\right]$ with countably many indeterminates $x_{n}, n \in Z^{+}$, consider the ideal $I=\left(x_{1}^{2}, x_{2}^{2}-x_{1}, x_{3}^{2}-x_{2}, \ldots\right)$ generated by $x_{1}^{2}$ and $x_{n+1}^{2}-x_{n}$ for each $n \in Z^{+}$. In the quotient ring $R=k\left[x_{1}, x_{2}, \ldots\right] / I$, the maximal ideal $M=\left(x_{1}, x_{2}, \ldots\right) / I$ of $R$ generated by all $\overline{x_{n}}=x_{n}+I, n \in Z^{+}$, is the unique maximal ideal of $R$. This is because, if $K$ is any maximal ideal of $R$, then $\bar{x}_{1}^{2}=0 \in K$ and so $\bar{x}_{1} \in K$ since $K$ is a prime ideal. Now $\bar{x}_{2}^{2}=\bar{x}_{1} \in K$ and so $\bar{x}_{2} \in K$. By induction, we obtain $\bar{x}_{n}^{2}=\bar{x}_{n-1} \in K$ and so $\bar{x}_{n} \in K$ for all $n \in Z^{+}$. Therefore $K=M$, as desired. Since $\bar{x}_{n}=\bar{x}_{n+1}^{2}$ for every $n \in Z^{+}$, we obtain $M=M^{2}$. So $\operatorname{Rad} M=M$ and hence $P(R)=M$. Since the ring $R / P(R)=R / M$ is a field (and so perfect), every $R$-module is Rad-supplemented (by Theorem 6.1). By [4, Lemma 28.3], $M=J(R)$ is not (left) $T$-nilpotent, and so $R$ is not a (left) perfect ring.

In [9], it is proved that the class of rings that are Rad-supplemented lies properly between the classes of semilocal rings and semiperfect rings. Recall that a ring $R$ is said to be a left duo ring if every left ideal of $R$ is a two-sided ideal. We shall characterize the left duo rings $R$ that are Radsupplemented left $R$-modules. Firstly, we need the following lemma:

LEMma 6.3. If $R$ is a left duo ring and $J, A, B$ are left ideals of $R$ such that $A+B=R$ and $A \cap B=J A \cap J B$, then $A \cap B=J(A \cap B)$.

Proof. Clearly $J(A \cap B) \leq A \cap B$. Conversely let $x \in A \cap B=J A \cap J B$. Since $A+B=R$, we have $a+b=1$ for some $a \in A$ and $b \in B$. Then $x=x a+x b$ and $x=\sum_{i \in I} s_{i} a_{i}=\sum_{i \in I^{\prime}} t_{i} b_{i}$ where $I, I^{\prime}$ are finite index sets, $a_{i} \in A$, $b_{i} \in B$ and $s_{i}, t_{i} \in J$. Now we have,

$$
x b=\sum_{i \in I} s_{i} a_{i} b \in J(A B) \text { and } x a=\sum_{i \in I^{\prime}} t_{i} b_{i} a \in J(B A) .
$$

Since $R$ is a left duo ring we have $A B \leq A \cap B$ and $B A \leq A \cap B$. So $x=x a+x b \in J(B A)+J(A B) \leq J(A \cap B)$. Thus $A \cap B \leq J(A \cap B)$.

Theorem 6.4. If $R$ is a left duo ring such that $P(R)=0$, then $R$ is a $R a d$-supplemented left $R$-module if and only if $R$ is semiperfect.

Proof. If $R$ is semiperfect, then $R$ is a supplemented, and so a Rad-supplemented, left $R$-module. Conversely, suppose $R$ is a Radsupplemented left $R$-module. Then $R$ is semilocal and $R$ is an amply 
Rad-supplemented left $R$-module by [1, 2.2(3) and 2.6(2)]. Let $A^{\prime}$ be a left ideal of $R$. Since $R$ is an amply Rad-supplemented left $R$ module, $A^{\prime}$ has a Rad-supplement $B$ in $R$, and $B$ has a Rad-supplement $A \leq A^{\prime}$ in $R$. So $R=A^{\prime}+B=A+B, A \cap B \leq A^{\prime} \cap B \leq \operatorname{Rad} B$ and $A \cap B \leq \operatorname{Rad} A$. Thus $A \cap B=(\operatorname{Rad} A) \cap(\operatorname{Rad} B)$. Let $J=J(R)$. Then $A \cap B=J A \cap J B=J(A \cap B)$ by Lemma 6.3. Since $R$ is a semilocal ring, $\operatorname{Rad}(A \cap B)=J(A \cap B)$. Then $A \cap B$ is a Rad-torsion submodule of $R$ and so $A \cap B \leq P(R)=0$. This gives that $R=A \oplus B$. Therefore $J B \leq J \ll R$ implies that $\operatorname{Rad}(B)=J B \ll B$ since $B$ is a direct summand of $R$. Hence $B$ is a supplement of $A^{\prime}$ in $R$. This shows that $R$ is a supplemented left $R$-module and so $R$ is semiperfect (see [30, 42.6]).

THEOREM 6.5. For a left duo ring $R$, the following are equivalent:

(i) $R / P(R)$ is semiperfect;

(ii) the left $R$-module $R$ is Rad-supplemented;

(iii) every finitely generated free left $R$-module is Rad-supplemented;

(iv) every finitely generated left R-module is Rad-supplemented.

Proof. (ii) $\Rightarrow$ (iii) follows by $[1,2.3(2)]$. (iii) $\Rightarrow$ (iv) holds since every finitely generated module is an epimorphic image of a finitely generated free module and Rad-supplemented modules are closed under epimorphic images. (iv) $\Rightarrow$ (ii) is clear.

(i) $\Rightarrow$ (ii): Since the quotient ring $S=R / P(R)$ is semiperfect, $R / P(R)$ is a Rad-supplemented left $S$-module and so a Rad-supplemented left $R$ module. Then the left $R$-module $R$ is Rad-supplemented by Proposition 4.8.

(ii) $\Rightarrow$ (i): The factor module $R / P(R)$ is also a Rad-supplemented left $R$ module. So the ring $S=R / P(R)$ is a Rad-supplemented left $S$-module with $P(S)=0$ and so $S=R / P(R)$ is semiperfect by Theorem 6.4.

Note that all implications except $(i i) \Rightarrow(i)$ of Theorem 6.5 hold for any ring $R$, while the implication $(i i) \Rightarrow(i)$ raises the question whether a Radsupplemented ring $R$ with $P(R)=0$ is necessarily semiperfect.

\section{Rad-supplemented Modules over Dedekind Domains.}

Over Dedekind domains, divisible modules coincide with injective modules as in abelian groups. Note that for a module $M$ over a Dedekind domain $R, M$ is divisible if and only if $\operatorname{Rad} M=M$, and this holds if and only 
if $M$ is injective; see for example [2, Lemma 4.4]. This is the motivation for the definition of reduced modules in general. A module over a Dedekind domain is reduced if it has no nonzero divisible submodules. As in abelian groups (see for example [11, Theorem 21.3]), any module $M$ over a Dedekind domain possesses a unique largest divisible submodule $D$ and $M=D \oplus C$ for a reduced submodule $C$ of $M$ (see [16, Theorem 8]); this $D$ is called the divisible part of $M$. Following the terminology in abelian groups, an $R$-module $M$ over a Dedekind domain is said to be bounded if $r M=0$ for some nonzero $r \in R$.

The structure of supplemented modules over Dedekind domains is completely determined in [32]:

Theorem 7.1 [32, Theorem 2.4. and Theorem 3.1]. Let $R$ be a Dedekind domain with quotient field $K \neq R$. Let $M$ be an $R$-module.

(i) Suppose $R$ is a local Dedekind domain, that is, a discrete valuation ring (DVR) with the unique prime element $p$. Then $M$ is supplemented if and only if $M \cong R^{a} \oplus K^{b} \oplus(K / R)^{c} \oplus B$ for some $R$-module $B$, where $a, b$, c are nonnegative integers and $p^{n} B=0$ for some integer $n>0$.

(ii) Suppose $R$ is non local. Then $M$ is supplemented if and only if $M$ is torsion and every primary component of $M$ is a direct sum of an artinian submodule and a bounded submodule.

Part (i) of the above theorem for Rad-supplemented modules is obtained as follows:

THeorem 7.2. Let $R$ be a DVR with quotient field $K \neq R$, and $p$ be the unique prime element. Then $M$ is Rad-supplemented if and only if $M \cong R^{a} \oplus K^{(I)} \oplus(K / R)^{(J)} \oplus B$ for some R-module $B$, where a is a nonnegative integer, $I, J$ are arbitrary index sets and $p^{n} B=0$ for some integer $n 0$.

Proof. $\quad(\Rightarrow)$ : If $M_{1}$ is the divisible part of $M$, then there exists a reduced submodule $M_{2}$ of $M$ such that $M=M_{1} \oplus M_{2}$. Since $M_{2}$ is also Radsupplemented, it is coatomic by Theorem 4.6. Then by [32, Lemma 2.1], $M_{2}=R^{a} \oplus B$, for some nonnegative integer $a$ and a bounded module $B$. Since $M_{1}$ is divisible, $M_{1} \cong K^{(I)} \oplus(K / R)^{(J)}$ for some index sets $I$ and $J$ (see [16, Theorem 7]).

$(\Leftarrow)$ : The module $N=K^{(I)} \oplus(K / R)^{(J)}$ is divisible, and so $\operatorname{Rad} N=N$. Then $N$ is Rad-supplemented by Proposition 4.5. By Theorem 7.1, the 
module $R^{a} \oplus B$ is supplemented, and hence Rad-supplemented. Therefore the direct sum $R^{a} \oplus K^{(I)} \oplus(K / R)^{(J)} \oplus B$ is Rad-supplemented.

Over commutative Noetherian rings we have:

Proposition 7.3. Let $R$ be a commutative noetherian ring and $M$ be a reduced $R$-module. Then $M$ is Rad-supplemented if and only if $M$ is supplemented.

Proof. Suppose $M$ is Rad-supplemented. Then $M$ is coatomic by Theorem 4.6, and so every submodule of $M$ is coatomic by [33, Lemma 1.1] since $R$ is a commutative noetherian ring. Let $U$ be a submodule of $M$ and $V$ be a Rad-supplement of $U$ in $M$. Then $V$ is coatomic, and so $U \cap V$ $\leq \operatorname{Rad} V \ll V$. Thus $V$ is a supplement of $U$ in $M$. The converse is clear.

Since the structure of supplemented modules is known by Theorem 7.1, it is enough to characterize Rad-supplemented modules in terms of supplemented modules. Note that for an $R$-module $M$ where $R$ is a Dedekind domain, $P(M)$ equals the divisible part of $M$.

Theorem 7.4. Let $R$ be a Dedekind domain and $M$ be an $R$-module. Then $M$ is Rad-supplemented if and only if $M / P(M)$ is (Rad-)supplemented.

Proof. Since $R$ is a Dedekind domain, $M$ has a decomposition as $M=P(M) \oplus N$ for some reduced submodule $N$ of $M$. If $M$ is Rad-supplemented, then $N \cong M / P(M)$ is also Rad-supplemented. Since $N$ is reduced, $N$ is supplemented by Proposition 7.3. Conversely, suppose $N \cong M / P(M)$ is Rad-supplemented. By Proposition 4.5-(ii), the submodule $P(M)$ is already Rad-supplemented. Therefore $M=P(M) \oplus N$ is Rad-supplemented as a sum of two Rad-supplemented modules.

These characterizations can be used to give examples of Rad-supplemented modules which are not supplemented.

ExAmple 7.5. Let $R$ be a Dedekind domain with quotient field $K \neq R$. The $R$-module $M=K^{(I)}$ is Rad-supplemented for every index set $I$. If $R$ is a local Dedekind domain (i.e. a DVR), then $M$ is supplemented only when $I$ is finite. If $R$ is a non-local Dedekind domain, then $M$ is not supplemented for every index set $I$, since $M$ is not torsion. 
Acknowledgments. The authors would like to thank Rafail Alizade who is the $\mathrm{PhD}$ advisors of the first two authors and who suggested the relative homological algebra approach to some problems related with complements and supplements and who has introduced the coneat concept. The authors would also like to thank the translators of the articles of Helmut Zöschinger from German to English: Rafail Alizade, Dilek Pusat-Yilmaz, O. Can Hatipoğlu and S. Eylem Erdoğan Toksoy. We would also like to express our thanks to Helmut Zöschinger for his excellent check of these translations. The authors are also grateful to John Clark for making valuable suggestions about our work. The authors also thank the referee for the corrections and comments.

\section{REFERENCES}

[1] K. Al-TAKhman - C. Lomp - R. WisBaueR, $\tau$-complemented and $\tau$-supplemented modules, Algebra Discrete Math., 3 (2006), pp. 1-16.

[2] R. Alizade - G. Bilhan - P. F. Smith, Modules whose maximal submodules have supplements, Comm. Algebra, 29 (6) (2001), pp. 2389-2405.

[3] R. Alizade - E. Mermut, The inductive closure of supplements, Journal of the Faculty of Science Ege University, 27 (2004), pp. 33-48.

[4] F. W. ANdERson - K. R. Fuller, Rings and Categories of Modules, Springer, New-York, 1992.

[5] G. AzUMAYA, A characterization of semi-perfect rings and modules, In "Ring Theory," Proc. Biennial Ohio-Denison Conf., World Scientific Publ. (Singapore, 1992) pp. 28-40.

[6] L. BICAN - T. KEPKA - P. NĚMEc, Rings, modules, and preradicals, volume 75 of Lecture Notes in Pure and Applied Mathematics, Marcel Dekker Inc., New York, 1982.

[7] P. E. BLAND, Topics in torsion theory, volume 103 of Mathematical Research, Wiley-VCH Verlag Berlin GmbH, Berlin, 1998.

[8] D. BuchsBAum, A note on homology in categories, Ann. of Math., 69 (1) (1959), pp. $66-74$.

[9] E. BÜYÜKAȘTK - C. LOMP, On a recent generalization of semiperfect rings, Bull. Aust. Math. Soc., 78 (2) (2008), pp. 317-325.

[10] J. Clark - C. Lomp - N. VANaJA - R. Wisbauer, Lifting modules, Frontiers in Mathematics. Birkhäuser Verlag, Basel, 2006. Supplements and projectivity in module theory.

[11] L. Fuchs, Infinite Abelian Groups, volume 1. Academic Press, New York, 1970.

[12] A. I. Generalov, The w-cohigh purity in a category of modules, Math. Notes, 33 (5-6) (1983), pp. 402-408. Translated from Russian from Mat. Zametki, 33 (5) (1983), pp. 785-796.

[13] J. S. GoLan, Torsion theories, volume 29 of Pitman Monographs and Surveys in Pure and Applied Mathematics, Longman Scientific Technical, Harlow, 1986. 
[14] D. K. Harrison - J. M. Irwin - C. L. Peercy - E. A. Walker, High extensions of abelian groups, Acta Math. Acad. Sci. Hunger., 14 (1963), pp. 319-330.

[15] K. HondA, Realism in the theory of abelian groups I, Comment. Math. Univ. St. Paul, 5 (1956), pp. 37-75.

[16] I. KAPLANSKY, Modules over Dedekind rings and valuation rings, Trans. Amer. Math. Soc., 72 (1952), pp. 327-340.

[17] F. KASCH, Modules and rings, volume 17 of London Mathematical Society Monographs. Academic Press Inc. [Harcourt Brace Jovanovich Publishers], London, 1982. Translated from the German and with a preface by D. A. R. Wallace.

[18] T. Y. LAM, A first course in noncommutative rings, volume 131 of Graduate Texts in Mathematics, Springer-Verlag, New York, second edition, 2001.

[19] C. Lomp, On semilocal modules and rings, Comm. Algebra, 27 (4) (1999), pp. 1921-1935.

[20] S. Maclane, Homology. Springer-Verlag, Berlin-Göttingen-Heidelberg, 1963.

[21] E. Mermut, Homological Approach to Complements and Supplements, $\mathrm{PhD}$ thesis, Dokuz Eylül University, The Graduate School of Natural and Applied Sciences, Izmir/Turkey, 2004.

http:/www.fbe.deu.edu.tr/ALL_FILES/Tez_Arsivi/2004/DR-t403.pdf.

[22] A. P. Mishina - L. A. SkornyaKov, Abelian groups and modules, volume 107 of American Mathematical Society Translations. Ser. 2. American Mathematical Society, Providence, R. I., 1976. Translated from Russian from Abelevy gruppy i moduli, Izdat. Nauka, Moscow (1969).

[23] S. NAKAHARA, On a generalization of semiperfect rings, Osaka J. Math., 20 (1) (1983), pp. 43-50.

[24] S. ÖzDEMIR, Neat and coneat subgroups, M. Sc. Thesis, Dokuz Eylül University, The Graduate School of Natural and Applied Sciences, Izmir/ Turkey, 2007.

http://www.fbe.deu.edu.tr/ALL_FILES/Tez_Arsivi/2007/YL_t2256.pdf.

[25] E. G. Sklyarenko, Relative homological algebra in categories of modules, Russian Math. Surveys, 33 (3) (1978), pp. 97-137. Traslated from Russian from Uspehi Mat. Nauk, 33, no. 3 (201), (1978), pp. 85-120.

[26] Bo T. Stenström, Rings of quotients, Springer-Verlag, New York, 1975. Die Grundlehren der Mathematischen Wissenschaften, Band 217, An introduction to methods of ring theory.

[27] Bo T. Stenström, High submodules and purity, Arkiv för Matematik, 7 (11) (1967), pp. 173-176.

[28] Bo T. Stenström, Pure submodules, Arkiv för Matematik, 7 (10) (1967), pp. 159-171.

[29] Y. WANG - N. Ding, Generalized supplemented modules, Taiwanese J. Math., 10 (6) (2006), pp. 1589-1601.

[30] R. Wisbauer, Foundations of Module and Ring Theory, Gordon and Breach, Reading, 1991.

[31] W. XuE, Characterization of semiperfect and perfect rings, Publicacions Matematiques, 40 (1) (1996), pp. 115-125.

[32] H. ZöschINGER, Komplementierte Moduln über Dedekindringen, J. Algebra, 29 (1974), pp. 42-56.

[33] H. Zöschinger, Koatomare Moduln, Math. Z., 170 (3) (1980), pp. 221-232.

Manoscritto pervenuto in redazione il 7 gennaio 2010. 